\title{
Automatic Spacecraft Docking Using Computer Vision-Based Guidance and Control Techniques
}

\author{
Chi-Chang J. Ho* and N. Harris McClamroch $\dagger$ \\ University of Michigan, Ann Arbor, Michigan 48109
}

\begin{abstract}
An innovative approach to automatic spacecraft docking using a computer vision-based control system is introduced. Precision control of the relative spacecraft velocity is required to achieve "soft" docking with a docking platform on a space station or on another spacecraft. We propose use of a computer vision system as a position and orientation sensor for obtaining feedback information used by guidance and control loops. $A$ camera, fixed to the spacecraft, tracks a standard rhombus mark fixed on the docking platform. Discrete-time position and orientation estimates of the spacecraft, relative to a coordinate frame fixed to the docking platform, are obtained by solving a constrained nonlinear least-squares problem and are used by the spacecraft feedback control loops. The accuracy of the computer vision estimates improves as the relative range decreases, thereby providing improved feedback information when it is most critical. Feedback control loops for the spacecraft, using three pairs of gas jet thrusters, are suggested that keep the camera always pointed at the rhombus mark and that perform precise control of the spacecraft range to achieve soft docking. The interactions between the vision system and the control systems are emphasized. Computer simulations of an integrated docking system verify the practical feasibility of this proposed automatic docking approach.
\end{abstract}

\section{Introduction}

A VARIETY of space missions require docking of spacecraft. ${ }^{1-4}$ Docking systems are usually classified according to their approach speed. Docking between a spacecraft and a space station or between two spacecraft is called "soft" if the final relative speed along their relative range direction is low. ${ }^{2}$ Precision control of spacecraft relative velocity and alignment is required during the approach phase to minimize docking contact forces.

A completely automatic spacecraft docking system is proposed using a computer vision system and a docking control system. Computer vision is a new technology that has not been exploited for many aerospace applications. The computer vision system serves as an accurate sensing device for estimation of spacecraft three-dimensional position and orientation relative to coordinates fixed at a standard rhombus mark on the docking platform. ${ }^{5,6}$ This approach compares favorably with other indirect or direct sensing techniques based on use of inertial navigation, laser, infrared, radar, or the global positioning system (GPS).

The docking control system consists of guidance and control loops that use these position and orientation estimates from the computer vision system to control the spacecraft sixdegree-of-freedom motion to achieve the spacecraft docking requirements. Rotational velocity estimation and attitude control loops, based on the three-dimensional orientation estimates obtained from the computer vision system, are developed to keep the camera pointed at the rhombus mark. Translational velocity estimation and control loops, based on the three-dimensional position estimates obtained from the computer vision system, are developed to control the relative translational motion between the spacecraft and the space station; the desired translational motion is defined according to current NASA Space Shuttle policy, ${ }^{7}$ which dictates that the spacecraft relative approach velocity with respect to the docking platform should be maintained at a value that is proportional to the relative range. The feedback control loops are assumed to be implemented using three pairs of gas jet

Received June 3, 1991; revision received March 3, 1992; accepted for publication March 26, 1992. Copyright (C) 1992 by the American Institute of Aeronautics and Astronautics, Inc. All rights reserved.

*Department of Aerospace Engineering. Member AIAA.

$\uparrow$ Professor, Department of Aerospace Engineering. Member AIAA. thrusters to simultaneously control the rotational and translational motion of the spacecraft.

Computer simulations of an integrated spacecraft computer vision and docking control system are described under a specific set of assumptions; the effects of image quantization and sampling, and the effects of orbital motion, are included. The simulation results demonstrate the technical feasibility of this proposed approach.

\section{Formulation of an Integrated Spacecraft Docking System}

In a previous paper, ${ }^{6}$ we investigated a computer vision system for obtaining estimates of spacecraft three-dimensional position and orientation. In this paper, we formulate an integrated spacecraft docking system based on the computer vision position and orientation estimates; these position and orientation estimates are obtained periodically to allow for the computer vision computations. Sampled data feedback control loops for the spacecraft are developed based on 1) zero-orderhold extrapolations of the position and orientation estimates between computer vision update times, and 2) translational and rotational velocity estimates obtained from observers which depend on the discrete-time position and orientation extrapolations. The computer vision processing time is relatively large $(0.5 \mathrm{~s}$ in our simulations), but docking errors that occur during the times between computer vision updates are corrected by the feedback control loops. The block diagram of the integrated spacecraft docking system is in Fig. 1.

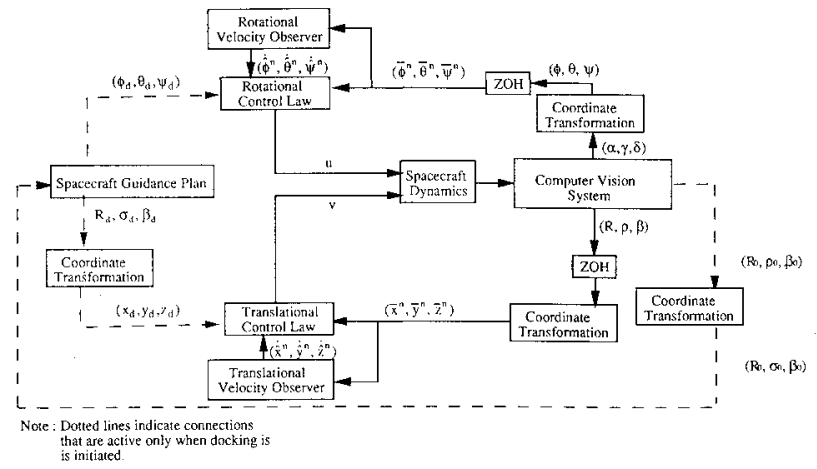

Fig. 1 Integrated docking system. 


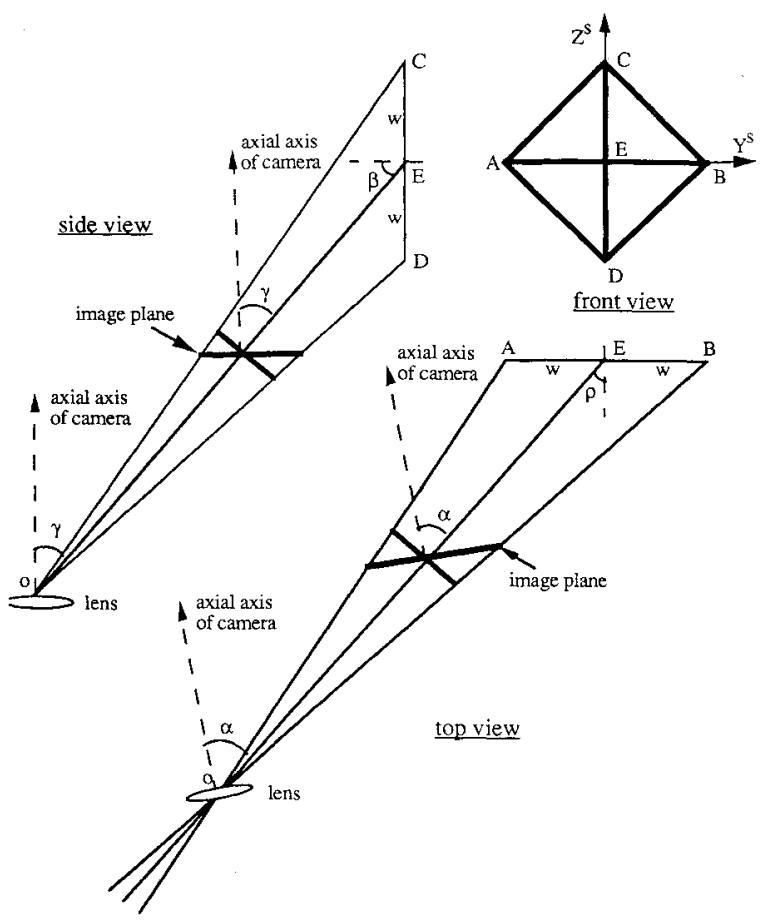

Fig. 2 Three-dimensional geometry of rhombus mark and camera.

The emphasis of this paper is on integration of the computer vision and the control loops in order to achieve the soft docking objective. Consequently, we make the following assumptions that somewhat simplify the details of the development.

1) The spacecraft is a rigid body with three pairs of gas jet thrusters mounted along the principal axes of the spacecraft to control the translational motion of the spacecraft and the rotational motion of the spacecraft. ${ }^{9}$

2) A pinhole camera is rigidly mounted on the spacecraft. A rhombus mark fixed on the docking platform of the space station is always in the field of view of the camera.

3) In the design of the feedback control loops, we assume that the space station provides an inertial reference. Of course, this is only a crude approximation which is satisfied if the total time to complete the docking maneuver is substantially shorter than the orbital period of the space station. Thus, orbital effects can be viewed as perturbations to the relative spacecraft and space station motion; these effects are ignored in the design of the spacecraft control loops.

\section{A. Computer Vision System}

The computer vision system serves as a sensor for the relative position and orientation between the spacecraft and the space station. The detailed development of the computer vision system is described elsewhere, ${ }^{6}$ a brief summary of these results is given here.

It is assumed that the image of the rhombus mark on the space station is characterized by the position of its four corners or feature points, as shown in Fig. 3. In particular, measurements of the location of the four feature points on the image plane provides eight measurement values. These eight measurement values $\left(X_{i}, Y_{i}\right), i=1,2,3,4$, depend on the relative geometry (see Fig. 2) of the camera and the rhombus mark according to the following expressions: ${ }^{6}$

$$
\begin{aligned}
& X_{1}=x_{c}+\frac{f w \cos \rho \cos \delta}{R \cos \alpha+w \sin (\rho+\alpha)}+n_{1} \\
& Y_{1}=y_{c}+\frac{f w \cos \rho \sin \delta}{R \cos \alpha+w \sin (\rho+\alpha)}+n_{2} \\
& X_{2}=x_{c}-\frac{f w \cos \rho \cos \delta}{R \cos \alpha-w \sin (\rho+\alpha)}+n_{3}
\end{aligned}
$$

$$
\begin{aligned}
& Y_{2}=y_{c}-\frac{f w \cos \rho \sin \delta}{R \cos \alpha-w \sin (\rho+\alpha)}+n_{4} \\
& X_{3}=x_{c}-\frac{f w \cos \beta \sin \delta}{R \cos \gamma+w \sin (\beta+\gamma)}+n_{5} \\
& Y_{3}=y_{c}+\frac{f w \cos \beta \cos \delta}{R \cos \gamma+w \sin (\beta+\gamma)}+n_{6} \\
& X_{4}=x_{c}+\frac{f w \cos \beta \sin \delta}{R \cos \gamma-w \sin (\beta+\gamma)}+n_{7} \\
& Y_{4}=y_{c}-\frac{f w \cos \beta \cos \delta}{R \cos \gamma-w \sin (\beta+\gamma)}+n_{8}
\end{aligned}
$$

Here $(\rho, \beta, R)$ are relative camera position parameters and $(\alpha, \gamma, \delta)$ are relative camera orientation parameters; $\left(x_{c}, y_{c}\right)$ are the coordinates of the center of the image of the rhombus mark on the image plane; $n_{i}, i=1, \ldots, 8$, are measurement errors due to quantization resolution on the image plane. The constant $f$ is the known focal length of the camera, and the constant $w$ is the known semidiagonal length of the rhombus mark.

Two geometric constraint equations that must be satisfied are

$$
\begin{aligned}
& f \tan \alpha=\sqrt{x_{c}^{2}+y_{c}^{2}} \cos \left[\tan ^{-1}\left(y_{c} / x_{c}\right)-\delta\right] \\
& f \tan \gamma=\sqrt{x_{c}^{2}+y_{c}^{2}} \sin \left[\tan ^{-1}\left(y_{c} / x_{c}\right)-\delta\right]
\end{aligned}
$$

Estimates of the relative position and relative orientation of the spacecraft, plus an estimate of the coordinates of the center of the image of the rhombus mark on the image plane, are obtained by solving a nonlinear constrained least-squares problem, ${ }^{6}$ i.e., minimize the sum of the squares of the residuals defined by Eqs. (1-8) subject to satisfaction of the constraint Eqs. (9-10). Additional details about the algorithm used in obtaining these position and orientation estimates and the properties of these estimates are available. ${ }^{6}$

It is also possible to express the estimates of the position of the spacecraft with respect to the docking platform in terms of spherical coordinates $(R, \beta, \sigma)$, where the spherical coordinates angle $\sigma$ is related to the camera angle $\rho$ and the angle $\beta$ by

$$
\sigma=\sin ^{-1}(\sin \rho / \cos \beta)
$$

or in terms of Cartesian coordinates $(x, y, z)$ where

$$
x=R \cos \beta \cos \sigma, \quad y=R \cos \beta \sin \sigma, \quad z=-R \sin \beta
$$

The relative orientation of the spacecraft with respect to the space station can also be expressed in terms of Euler angles $(\phi, \theta, \psi)$ for roll, pitch, yaw; in particular the Euler angles can be expressed in terms of the camera orientation parameters as

$$
\phi=\delta, \quad \theta=\gamma+\beta, \quad \psi=\sin ^{-1}\left[\frac{\sin (\alpha+\rho)}{\cos (\gamma+\beta)}\right]
$$

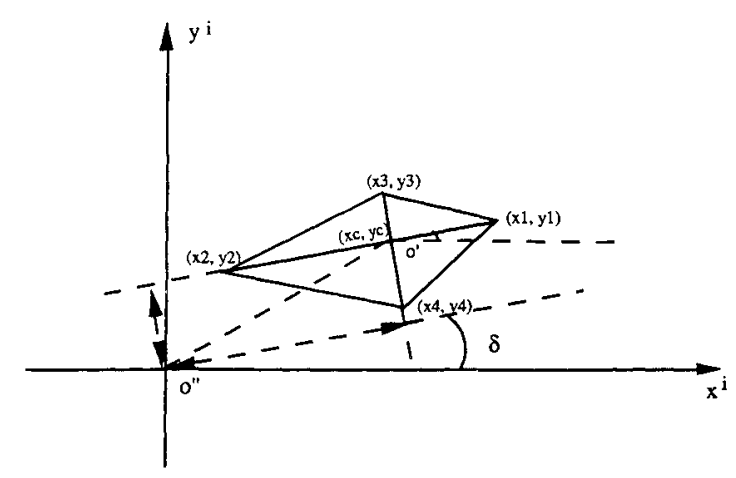

Fig. 3 Projection of rhombus mark on the image plane. 
The main conclusion is that the vision system provides discrete-time estimates of the position and orientation of the spacecraft relative to the coordinate frame fixed to the rhombus mark. When docking is initiated, initial condition estimates obtained from the computer vision system for the relative position and orientation explicitly determine the desired docking maneuver and are used to initialize the observers. The initial position estimate of the spacecraft with respect to the docking platform, obtained from the computer vision system, is given by $\left(R_{0}, \sigma_{0}, \beta_{0}\right)$, expressed in spherical coordinates; these initial data are used to define the specific docking maneuver to be achieved. The initial orientation of the spacecraft with respect to the docking platform, obtained from the computer vision estimates, is given by $\left(\phi_{0}, \theta_{0}, \psi_{0}\right)$, expressed in terms of Euler angles. This initial data is used to initialize the observers used in the spacecraft control loops.

To achieve adequate accuracy for the computer vision position and orientation estimates, adaptive zoom is required to adjust the image size on the camera's image plane. The size of the image of the rhombus mark on the image plane is proportional to $w f / R$. If the range $R$ is large, then the image size is small, so that adaptive zoom is required to adjust the focal length to enlarge the image size. An adaptive zoom magnification rule is defined by specifying the focal length $f$ as an a priori specified function of the estimated range; our experience is that a very crude adaptive zoom rule suffices.

\section{B. Rotational Velocity Estimation and Attitude Control Loops}

In this section, we describe the rotational control loops in Fig. 1. A nonlinear rotational velocity observer which asymptotically reconstructs the spacecraft angular velocities based only on zero-order-hold extrapolations of the spacecraft orientation estimates from the computer vision system is developed in detail. A sampled data nonlinear rotational control law uses zero-order-hold extrapolations of the discrete-time spacecraft orientation estimates obtained from the computer vision system and rotational velocity estimates from the nonlinear rotational observer to maintain the desired constant orientation between the spacecraft and the space station, namely,

$$
\phi_{d}(t)=0, \quad \theta_{d}(t)=\beta_{0}, \quad \psi_{d}(t)=\alpha_{0}, \quad t \geq 0
$$

Our nonlinear observer-based controller is based on dynamic equations for the rotational motion of the spacecraft expressed in terms of Euler angles $(\psi, \theta, \phi)$ for yaw, pitch, and roll; the spacecraft kinematic equations, expressed in a coordinate frame defined by the spacecraft principal axes, are ${ }^{9}$

$$
\omega=J(q) \dot{q}
$$

where $\omega=\left[\begin{array}{lll}\omega_{1} & \omega_{2} & \omega_{3}\end{array}\right]^{T}$ denotes the angular velocity of the spacecraft in the body frame, $q=\left[\begin{array}{lll}\phi & \theta & \psi\end{array}\right]^{T}$ is the attitude vector, and

$$
J(q)=\left[\begin{array}{ccc}
1 & 0 & -\sin \theta \\
0 & \cos \phi & \sin \phi \cos \theta \\
0 & -\sin \phi & \cos \phi \cos \theta
\end{array}\right]
$$

Let $J^{*}$ denote the diagonal inertia matrix of the spacecraft in the coordinate frame defined by its principal axes. The Euler equations for the dynamics of the spacecraft are given by ${ }^{9}$

$$
J^{*} \dot{\omega}=S(\omega) J^{*} \omega+B u
$$

where

$$
S(\omega)=\left[\begin{array}{ccc}
0 & \omega_{3} & -\omega_{2} \\
-\omega_{3} & 0 & \omega_{1} \\
\omega_{2} & -\omega_{1} & 0
\end{array}\right]
$$

is a skew symmetric matrix and $B$ is a constant nonsingular input matrix and $u$ is the vector of torques along the three spacecraft principal axes supplied by the thrusters. Substituting Eq. (12) into Eq. (13), we obtain equations in terms of Euler angles

$$
J^{T}(q) J^{*} J(q) \ddot{q}+C(q, \dot{q}) \dot{q}=J^{T}(q) B u
$$

where $C(q, \dot{q})$ is linear in $\dot{q}$.

Although a linear controller could be designed based on linearization of the above equations, our research has indicated that substantial improvement in the size of the domain of attraction is obtained by using a nonlinear observer-based controller. This is a critical issue since serious consequences arise if the orientation of the spacecraft is such that the rhombus mark fixed on the space station is not in the field of view of the camera.

Consequently, the proposed controller is nonlinear. Our approach follows the development in the literature ${ }^{8,10}$ Equations for the nonlinear rotational observer are

$$
\begin{gathered}
\dot{\hat{x}}_{1}=\hat{x}_{2}+k_{o}\left(\bar{q}-\hat{x}_{1}\right) \\
J^{T}(\bar{q}) J^{*} J(\bar{q}) \dot{\hat{x}}_{2}=-C\left(\bar{q}, \dot{\hat{x}}_{1}\right) \dot{\hat{x}}_{1}+K_{o}\left(\bar{q}-\hat{x}_{1}\right)+J^{T}(\bar{q}) B u
\end{gathered}
$$

where $k_{o}$ is a positive constant and $K_{o}$ is a symmetric, positive definite matrix and $\bar{q}=(\bar{\phi}, \bar{\theta}, \bar{\psi})$ denotes the zero-order-hold extrapolations of the discrete-time orientation estimates obtained from the computer vision system. The initial orientation angles for the rotational observer are the initial computer vision orientation estimates

$$
\hat{\phi}_{1}(0)=\phi_{0}, \quad \hat{\theta}_{1}(0)=\theta_{0}, \quad \hat{\psi}_{1}(0)=\psi_{0}
$$

The initial orientation rates for the rotational observer are selected according to the desired rotational guidance commands, namely,

$$
\hat{\phi}_{2}(0)=0, \quad \hat{\theta}_{2}(0)=0, \quad \hat{\psi}_{2}(0)=0
$$

We want the spacecraft to track the desired constant attitude commands $q_{d}=\left(0, \beta_{0}, \sigma_{0}\right)$ so that we consider the following sampled data controller:

$$
u=J^{-T}(\bar{q})\left[-k_{C}\left(\bar{q}-q_{d}\right)-K_{C} \dot{\hat{x}}_{1}\right]
$$

where $k_{C}$ and $K_{C}$ are symmetric, positive definite matrices. The zero-order-hold orientation extrapolations $\bar{q}=(\bar{\phi}, \bar{\theta}, \bar{\psi})$ are obtained from the discrete-time computer vision orientation estimates and the angular velocity estimates $\dot{\hat{x}}_{1}=\left(\hat{\phi}_{2}, \hat{\theta}_{2}, \hat{\psi}_{2}\right)$ are obtained from the rotational observer.

Selection of specific values of the control gains in Eqs. (1516) and (19) can be made on the basis of linearization of the nonlinear rotational closed-loop equations about the indicated nominal operating point. ${ }^{10}$

\section{Translational Velocity Estimation and Control Loops}

In this section, we describe the translational control loops in Fig. 1. A linear translational velocity observer which asymptotically reconstructs the spacecraft translational velocities based only on zero-order-hold extrapolations of the spacecraft position estimates obtained from the computer vision system is developed. A sampled data linear translational controller uses zero-order-hold extrapolations of the discrete-time spacecraft position estimates obtained from the computer vision system and translational velocity estimates from the linear translational observer to track the desired relative translational motion between the spacecraft and the docking platform.

The desired relative translational motion between the spacecraft and the space station is defined using current NASA Space Shuttle docking specifications. ${ }^{7}$ This rule dictates that 
the relative approach velocity is along the range vector and is proportional to the relative range. If the initial range estimate of the spacecraft relative to the coordinate frame fixed on the docking platform is $R_{0}$, the desired range is

$$
R_{d}(t)=R_{0} e^{-\lambda t}
$$

where $\lambda>0$ is a docking proportionality constant.

It is also desired to maintain the spherical coordinate heading angles constant at their initial values, that is for $t \geq 0$

$$
\sigma_{d}(t)=\sigma_{0}, \quad \beta_{d}(t)=\beta_{0}
$$

where $\sigma_{0}, \beta_{0}$ are angle estimates when the docking maneuver is initiated.

We can transform this desired translational motion $\left(R_{d}(t), \sigma_{d}(t), \beta_{d}(t)\right)$ from spherical coordinates to relative Cartesian coordinates to obtain the desired spacecraft translational guidance commands

$$
\begin{array}{cc}
x_{d}(t)=R_{0} e^{-\lambda t} \cos \beta_{0} \cos \sigma_{0}, & t \geq 0 \\
y_{d}(t)=R_{0} e^{-\lambda t} \cos \beta_{0} \sin \sigma_{0}, & t \geq 0 \\
z_{d}(t)=-R_{0} e^{-\lambda t} \sin \beta_{0}, & t \geq 0
\end{array}
$$

Dynamic equations for the spacecraft translational motion relative to the docking platform are

$$
\begin{aligned}
& m \ddot{x}=v_{1} \\
& m \ddot{y}=v_{2} \\
& m \ddot{z}=v_{3}
\end{aligned}
$$

where $v_{1}, v_{2}, v_{3}$ are the thrust forces along the spacecraft principal axes supplied by the thrusters and $m$ is the spacecraft mass.

The spacecraft velocities are estimated based on computer vision position extrapolations using a translational observer. Equations for the linear translational observer are

$$
\begin{aligned}
& \dot{\hat{x}}_{1}=\hat{x}_{2}+k_{4}\left(\bar{x}-\hat{x}_{1}\right) \\
& \dot{\hat{y}}_{1}=\hat{y}_{2}+k_{5}\left(\bar{y}-\hat{y}_{1}\right) \\
& \dot{\hat{z}}_{1}=\hat{z}_{2}+k_{6}\left(\bar{z}-\hat{z}_{1}\right) \\
& m \dot{\hat{x}}_{2}=v_{1}+k_{1}\left(\bar{x}-\hat{x}_{1}\right) \\
& m \dot{\hat{y}}_{2}=v_{2}+k_{2}\left(\bar{y}-\hat{y}_{1}\right) \\
& m \dot{\hat{z}}_{2}=v_{3}+k_{3}\left(\bar{z}-\hat{z}_{1}\right)
\end{aligned}
$$

where $(\bar{x}, \bar{y}, \bar{z})$ are zero-order-hold extrapolations of the discrete-time position estimates obtained from the computer vision system. The initial position estimates of the translational observer are the initial position estimates obtained from the computer vision system

$$
\hat{x}_{1}(0)=x_{0}, \quad \hat{y}_{1}(0)=y_{0}, \quad \hat{z}_{1}(0)=z_{0}
$$

The initial velocities of the translational observer are selected according to the desired translational guidance commands, namely,

$$
\begin{gathered}
\hat{x}_{2}(0)=-\lambda R_{0} \cos \beta_{0} \cos \sigma_{0} \\
\hat{y}_{2}(0)=-\lambda R_{0} \cos \beta_{0} \sin \sigma_{0} \\
\hat{z}_{2}(0)=\lambda R_{0} \sin \beta_{0}
\end{gathered}
$$

A sampled data controller for the translational loops is

$$
\begin{aligned}
& v_{1}=m \ddot{x}_{d}+k_{10}\left(\dot{x}_{d}-\hat{x}_{2}\right)+k_{7}\left(x_{d}-\bar{x}\right) \\
& v_{2}=m \ddot{y}_{d}+k_{11}\left(\dot{y}_{d}-\hat{y}_{2}\right)+k_{8}\left(y_{d}-\bar{y}\right) \\
& v_{3}=m \ddot{z}_{d}+k_{12}\left(\dot{z}_{d}-\hat{z}_{2}\right)+k_{9}\left(z_{d}-\bar{z}\right)
\end{aligned}
$$

where $(\bar{x}, \bar{y}, \bar{z})$ are zero-order-hold extrapolations of the position estimates obtained from the computer vision system; the velocity estimates $\left(\hat{x}_{2}, \hat{y}_{2}, \hat{z}_{2}\right)$ are obtained from the translational observer.

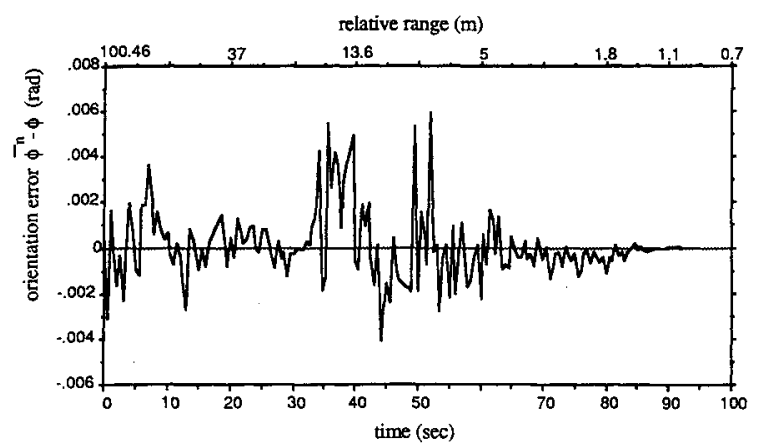

Fig. 4 Estimation errors in roll angle from computer vision system.

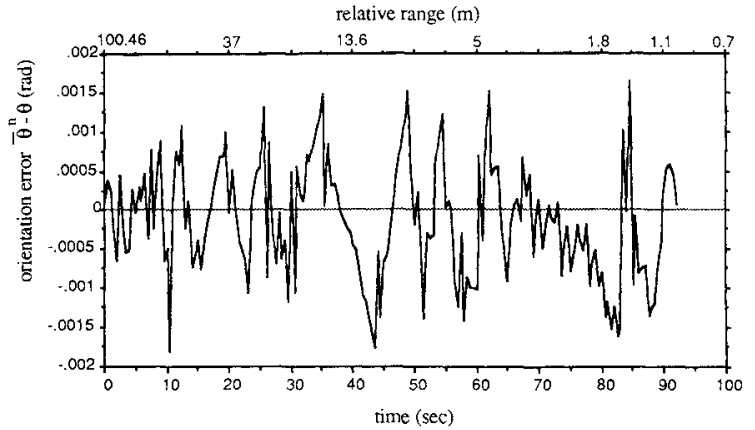

Fig. 5 Estimation errors in pitch angle from computer vision system.

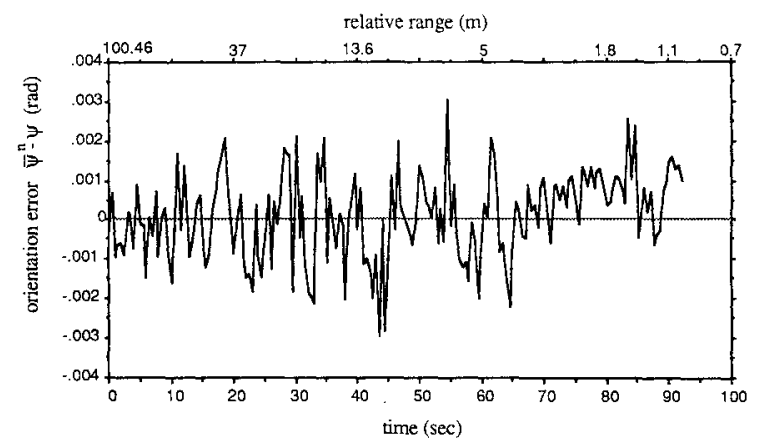

Fig. 6 Estimation errors in yaw angle from computer vision system.

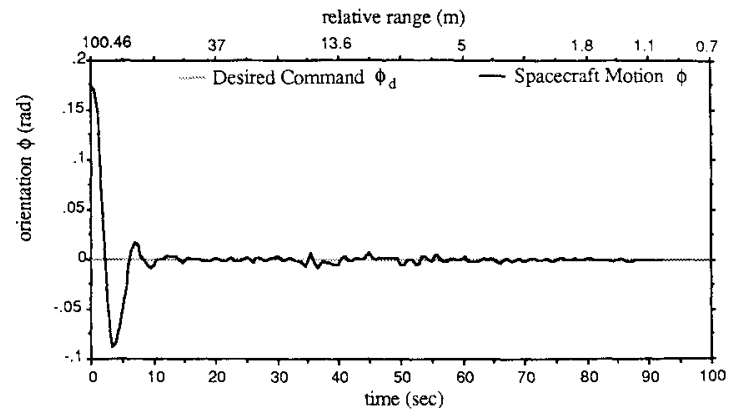

Fig. 7 Controlled roll motion. 
Selection of the specific values of the observer and controller gain matrices in Eqs. (28-32) and Eqs. (38-40) can be made using standard methods for the linear closed-loop translational loops. ${ }^{10}$

The control accelerations for the translational motion control loops can be transformed to the body frame to obtain the inputs for the spacecraft thrusters.

\section{Comments}

We now summarize the main features of the computer vision and feedback control loops, emphasizing the inherent interactions between the design issues for each. First, the computer vision system provides discrete-time estimates of the spacecraft position and orientation relative to the docking platform. The time between computer vision estimates is necessarily long due to the real-time computational requirements of the vision system. The computer vision system provides discrete-time estimates of spacecraft position and orientation so that observers must be constructed to obtain estimates of the spacecraft rotational and translational velocities; a sampled data observerbased controller can be constructed that depends on the sampled data obtained from the computer vision system. The accuracy of the position and orientation estimates of the spacecraft, obtained from the computer vision system, is weakly dependent on the pixel quantization of the image plane but it is strongly affected by the range; it has been found to be essential to include a simple adaptive zoom mechanism. Next, the design of the feedback control loops is influenced by the fact that they must be robust to initialization errors and to errors in the position and orientation estimates obtained from the computer vision system; they must also be able to compensate for the relatively long times between estimates obtained from the computer vision system and they must be able to compensate for orbital and other effects that have been ignored in the control loop designs. We have used sampled data controllers based on sampled data observers that reconstruct rotational and translational velocity estimates form the position and orientation estimates provided by the computer vision system; in the case of the rotational loop both the sampled data observer and the sampled data controller are nonlinear to provide the required robustness to keep the camera always pointed at the rhombus mark on the docking platform.

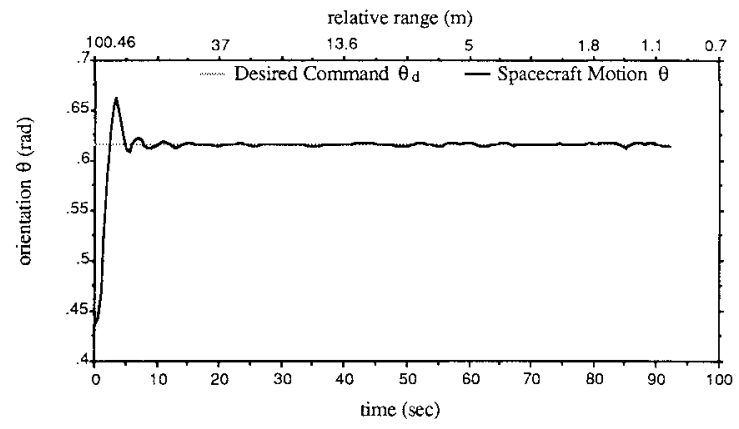

Fig. 8 Controlled pitch motion.

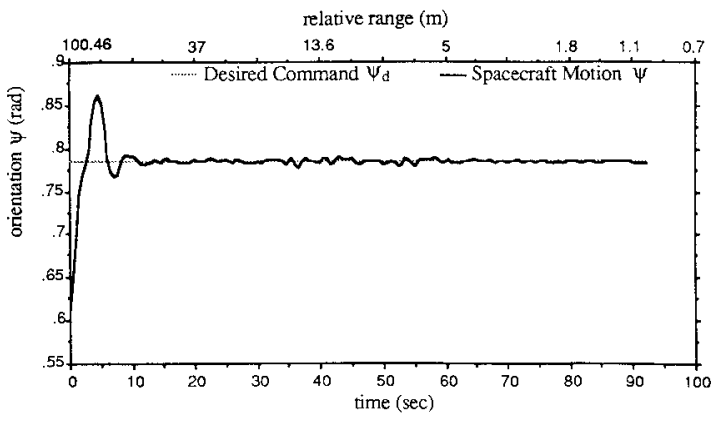

Fig. 9 Controlled yaw motion.
A detailed investigation of the closed-loop docking system, including the error effects due to the computer vision computations and extrapolations and the error effects due to orbital perturbations, is very complicated. However, if these error sources are ignored then an analytical investigation of the closed-loop docking system has been carried out. ${ }^{8,10}$ To summarize those results, ${ }^{8,10}$ it has been shown that the ideal rotational closed loop is asymptotically stable in the sense that

$$
\phi \rightarrow \phi_{d}, \quad \theta \rightarrow \theta_{d}, \quad \psi \rightarrow \psi_{d}, \quad \text { as } t \rightarrow \infty
$$

for all initial conditions in a domain of attraction that can be explicitly characterized. In addition, it has been shown that the ideal translational closed loop is asymptotically stable in the sense that

$$
x \rightarrow x_{d}, \quad y \rightarrow y_{d}, \quad z \rightarrow z_{d}, \quad \text { as } t \rightarrow \infty
$$

These theoretical results provide confidence that the proposed feedback control loops achieve the desired docking control objectives even in the case that realistic errors described above are present.

\section{Simulation of an Integrated Spacecraft Docking System}

In this section, we present representative digital computer simulations to verify the practical feasibility of this integrated docking system and to identify difficulties and restrictions on implementation of the system. Parameter values are selected to represent a simple, but realistic, case consistent with the assumptions indicated previously.

\section{A. Simulation Strategy}

1) The computational speed of the onboard vision computer is assumed to be sufficiently fast, so that the image-processing requirements and the computation of position and orientation estimates can be completed within $0.5 \mathrm{~s}$. Thus, the time between position and orientation estimates obtained from the computer vision system is taken as $0.5 \mathrm{~s}$. An adaptive zoom magnification rule is used to magnify the image at large range values; the adaptive rule is 1 ) if the range estimate satisfies $R>20 \mathrm{~m}$, the image magnification factor is 10 ; and 2) if the range estimate satisfies $R \leq 20 \mathrm{~m}$, the image magnification factor is 1 .

2) A translational docking policy using a " $5 \%$ rule," i.e., $\lambda=0.05$, is chosen. An initial range of $100 \mathrm{~m}$ requires a relatively short total docking maneuver time (about $100 \mathrm{~s}$ ).

3) We assume the following camera parameter values: the semidiagonal length of the rhombus mark is $w=2 \mathrm{~m}$, the focal length of the camera is $f=0.2 \mathrm{~m}$, the image plane size is $0.1 \times 0.1 \mathrm{~m}$, and the width of a pixel on the image plane is $0.00039 \mathrm{~m}$. All other parameters for the computer vision system are the same as in the simulation experiments reported elsewhere. ${ }^{6}$

4) The spacecraft is assumed to be a symmetric rigid body with three identical principal moments of inertia.

5) The rotational observer gains are selected so that the linearized rotational observer has a bandwidth of $0.89 \mathrm{~Hz}$; the rotational controller gains are selected so that the linearized closed loop for the rotational motion has a bandwidth of 0.22 $\mathrm{Hz}$. The translational observer gains are selected so that the translational observer has a bandwidth of $0.89 \mathrm{~Hz}$; the translational controller gains are selected so that the closed loop for the translational motion has a bandwidth of $0.22 \mathrm{~Hz}$.

6) The true initial position of the spacecraft relative to the space station is

$$
x_{0}=58 \mathrm{~m}, \quad y_{0}=58 \mathrm{~m}, \quad z_{0}=-58 \mathrm{~m}
$$

corresponding to an initial range of $R_{0}=100.46 \mathrm{~m}$ and angles $\sigma_{0}=45 \mathrm{deg}, \beta_{0}=35.26 \mathrm{deg}$. The true initial relative velocity of the spacecraft is assumed to be

$$
\dot{x}_{0}=\dot{y}_{0}=\dot{z}_{0}=0 \mathrm{~m} / \mathrm{s}
$$


The true initial orientation and orientation rates of the spacecraft are assumed to be

$$
\begin{gathered}
\phi_{0}=10 \mathrm{deg}, \quad \theta_{0}=25 \mathrm{deg}, \quad \psi_{0}=35 \mathrm{deg} \\
\dot{\phi}_{0}=\dot{\theta}_{0}=\dot{\psi}_{0}=0 \mathrm{deg} / \mathrm{s}
\end{gathered}
$$

7) According to the 5\% rule, the desired range command is

$$
R_{d}(t)=100.46 e^{-0.05 t}, \quad t \geq 0
$$

The desired translational commands used by the translational control loops, in Cartesian coordinates, are

$$
\begin{array}{ll}
x_{d}(t)=100.46 e^{-0.05 t} \cos \beta_{0} \cos \sigma_{0}, & t \geq 0 \\
y_{d}(t)=100.46 e^{-0.05 t} \cos \beta_{0} \sin \sigma_{0}, \quad t \geq 0 \\
z_{d}(t)=100.46 e^{-0.05 t} \sin \beta_{0}, \quad t \geq 0
\end{array}
$$

The desired orientation angles are based on initial conditions, obtained by the computer vision system; the desired orientation angles are

$$
\begin{gathered}
\sigma_{d}(t)=45 \mathrm{deg}, \quad \beta_{d}(t)=35.26 \mathrm{deg} \\
\rho_{d}(t)=35.26 \mathrm{deg}, \quad t \geq 0
\end{gathered}
$$

The desired rotational commands used by the rotational control loops are

$$
\phi_{d}(t)=0, \quad \theta_{d}(t)=35.26 \mathrm{deg}, \quad \psi_{d}(t)=45 \mathrm{deg}, \quad t \geq 0
$$

The desired roll command about the axial axis of the camera is

$$
\delta_{d}(t)=0, \quad t \geq 0
$$

8) To capture physical limits on the thrusters, a saturation limit of $2 \mathrm{~m} / \mathrm{s}^{2}(0.2 \mathrm{~g})$ is imposed on the controlled accelerations for the translational motion loops. The initial position of the translational observer obtained from computer vision estimates are

$$
\hat{x}_{0}=55.2 \mathrm{~m}, \quad \hat{y}_{0}=58.5 \mathrm{~m}, \quad \hat{z}_{0}=-58.5 \mathrm{~m}
$$

The initial translational velocity for the translational observer is

$$
\dot{\hat{x}}_{0}=-2.9 \mathrm{~m} / \mathrm{s}, \quad \dot{\hat{y}}_{0}=-2.9 \mathrm{~m} / \mathrm{s}, \quad \dot{\hat{z}}_{0}=2.9 \mathrm{~m} / \mathrm{s}
$$

9) To capture physical limits on the thrusters, a saturation limit of $5 \mathrm{deg} / \mathrm{s}^{2}$ is imposed on the controlled angular accelerations for the rotational motion loops. The initial conditions for the rotational observer obtained from computer vision estimates are

$$
\hat{\phi}_{0}=10.11 \mathrm{deg}, \quad \hat{\theta}_{0}=25.11 \mathrm{deg}, \quad \widehat{\psi}_{0}=35.12 \mathrm{deg}
$$

The initial rotational velocity for the rotational observer is

$$
\dot{\hat{\phi}}_{0}=\dot{\hat{\theta}}_{0}=\dot{\hat{\psi}}_{0}=0 \mathrm{deg} / \mathrm{s}
$$

10) The nominal orbit of the space station is assumed to have a 90-min period; hence the docking duration of $100 \mathrm{~s}$ corresponds to an orbital angle of $6.7 \mathrm{deg}$. The orbital effect on the simulation of the translational motion is ignored in the simulations. On the other hand, the orbital effect is included in the simulation of the relative rotational motion as a constant drift rate of $0.067 \mathrm{deg} / \mathrm{s}$ in the relative yaw angle between the spacecraft and the docking platform.

11) A stopping criterion for the spacecraft docking simulation is chosen when the relative range is reduced to $1 \mathrm{~m}$.
12) Simulation of the rotational motion loops is separated from the simulation of the translational motion loops. The only coupling between the rotational loops and the translational loops is through the computer vision system which supplies simultaneous position and orientation estimates. This coupling between the orientation estimates and the position estimates in the computer vision system is very weak so that independent simulations of the rotational motion and the translational motion is justified. To be consistent with the translational loops, the $5 \%$ rule for the desired translational motion is chosen to characterize the range effects in the simulation of the rotational loops.

\section{B. Simulation Results and Performance Evaluation}

The above implementation of the integrated docking control system has been simulated in Fortran on an Apollo Domain 4000 computer; additional details about the simulation are available. ${ }^{8}$ The following simulation results are typical of the many different simulations performed; they allow evaluation of performance in terms of the accuracy with which the docking mission is accomplished.

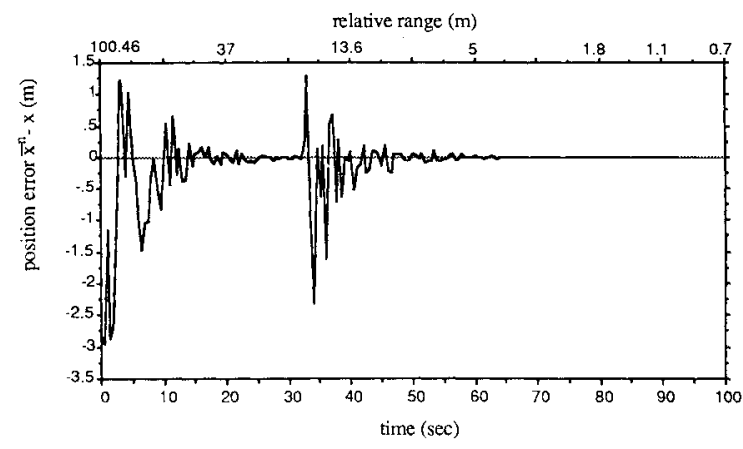

Fig. 10 Estimation errors in $x$ direction from computer vision system.

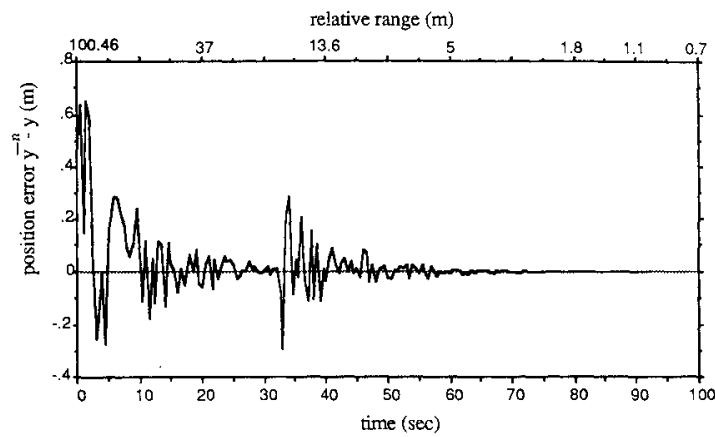

Fig. 11 Estimation errors in $y$ direction from computer vision system.

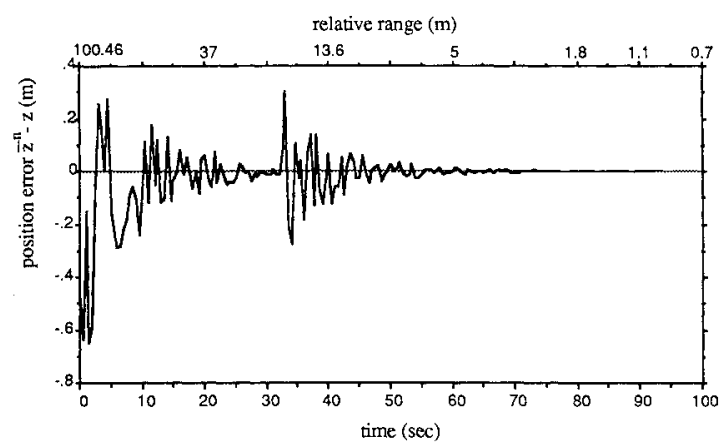

Fig. 12 Estimation errors in $z$ direction from computer vision system. 


\section{Rotational Velocity Estimation and Attitude Control Loop} Simulations

Figures $4-6$ show the angular position $(\phi, \theta, \psi)$ estimation errors from the computer vision system. It can be seen that there are minor increases in the estimation errors around $t=34 \mathrm{~s}$ since this is the time when the zoom magnification factor switches form 10 to 1 . Nevertheless, the estimation errors from the computer vision system are quite small. In particular, the computer vision estimation errors for pitch and yaw are very small (less than $0.115 \mathrm{deg}$ ), which is the accuracy limit of the computer vision system.

Figures 7-9 show the ability of the rotational control loops to regulate the orientation of the spacecraft about the desired attitude. It can be seen that the accuracy of the computer vision estimates does influence the control errors, the control accuracy improves as the range decreases. The initial spacecraft orientation was selected to differ considerably from the desired spacecraft docking orientation; it is seen from these figures that the nonlinear observer-based rotational controller is able to maintain control of the spacecraft to keep it pointed at the rhombus mark. Although orbital perturbations were included in the simulations as indicated, their effect on the accuracy of the rotational loops is negligible.

2. Translational Velocity Estimation and Control Loop Simulations

Figures 10-12 show the position estimation errors from the computer vision system. These translational estimation errors

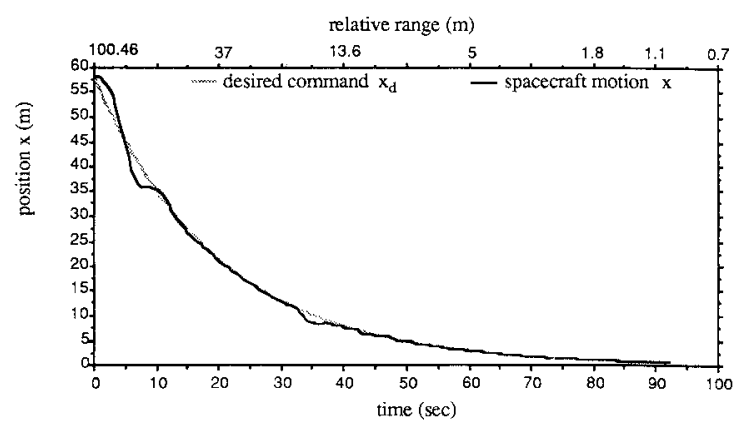

Fig. 13 Controlled $x$-direction motion.

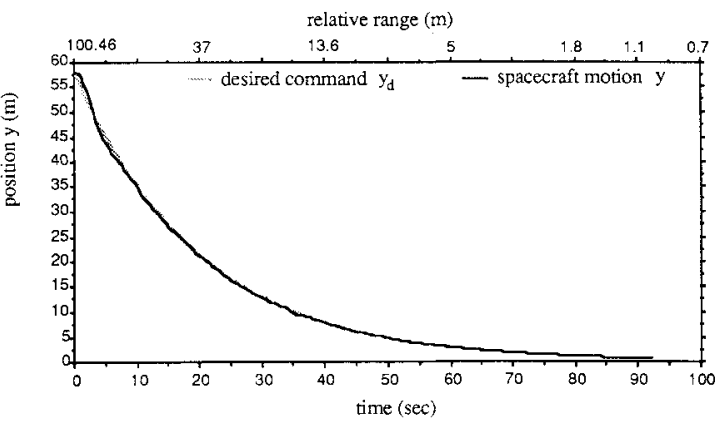

Fig. 14 Controlled $y$-direction motion.

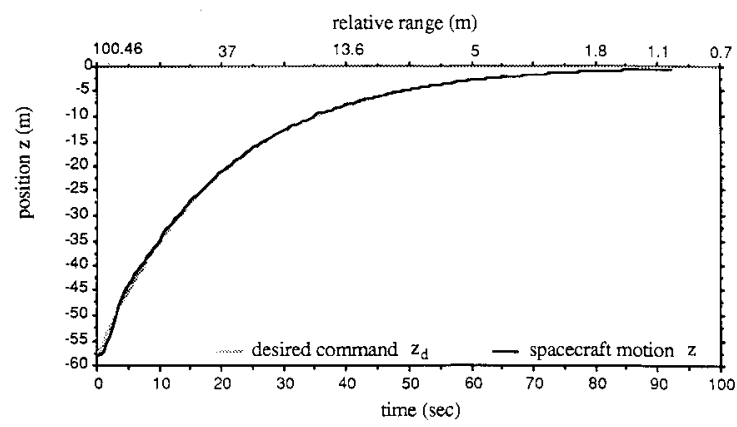

Fig. 15 Controlled $z$-direction motion. are significant when the spacecraft is far from the docking platform, but they are substantially decreased as the spacecraft approaches the docking platform. It can be seen that the position estimation errors also increase just after $t=34 \mathrm{~s}$ due to the change in the zoom magnification factor.

Figures $13-15$ indicate the desired and controlled translational motion from simulations of the translational control loops. Figures 16-18 show the translational control errors for the translational control loops. It is clear that the translational control errors are significant when the spacecraft is far from the docking platform, but they are substantially decreased as the spacecraft approaches the docking platform. The increase in translational estimation errors beginning at $t=34 \mathrm{~s}$ can be seen to cause an increase in the control errors in the translational loops just after $t=34 \mathrm{~s}$.

\section{Comments}

As is typical for computer vision systems, the estimation accuracy of the spacecraft orientation is considerably better than the estimation accuracy for the spacecraft position, the largest errors occurring in the range estimates. The computer vision errors are proportional to the effective range (range after zoom magnification adjustment). This is very clear from the translational loop simulations. Thus the control errors are also proportional to the effective range. The final translational velocity with which the spacecraft approaches the docking platform is very low and is essentially determined by the $5 \%$

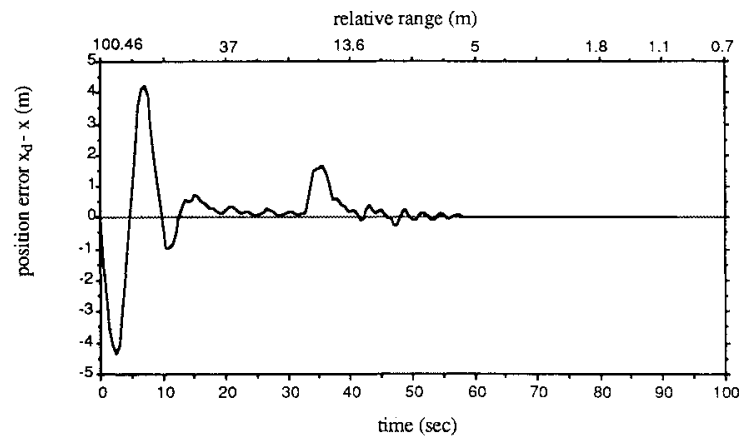

Fig. 16 Controlled $x$-direction errors.

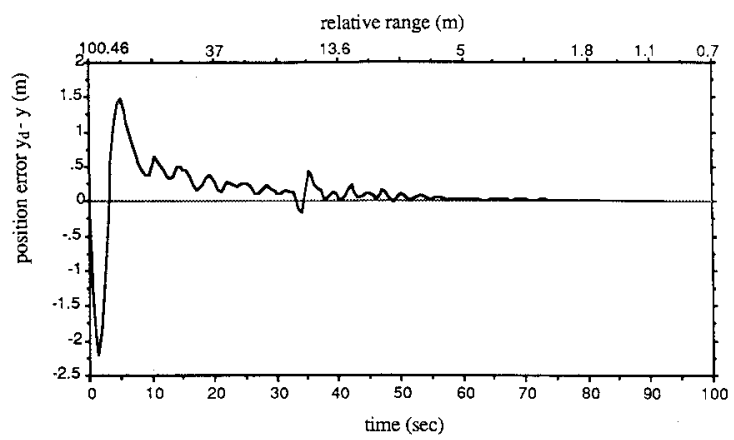

Fig. 17 Controlled $y$-direction errors.

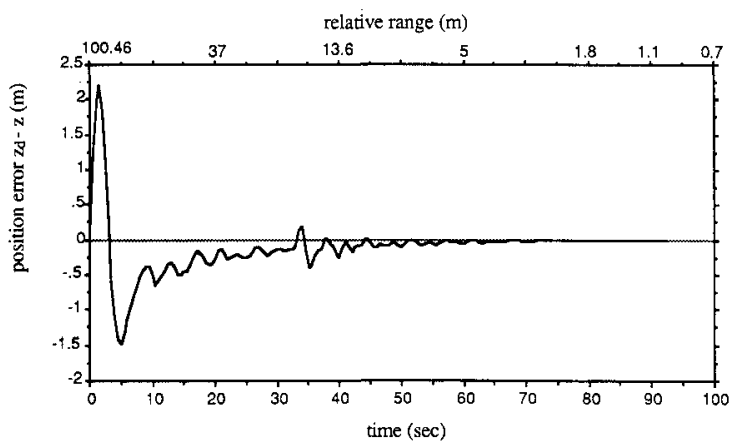

Fig. 18 Controlled $z$-direction errors. 
rule; the accuracy of the orientation estimates from the computer vision system during final approach is quite good, better than $0.115 \mathrm{deg}$. The influence of computer vision extrapolation and sampled data effects and orbital effects on the accuracy of the integrated docking system is negligible.

Two important points should be emphasized. The camera must have automatic, adaptive zoom to compensate for range effects on the image of the rhombus mark. If the range is large, the zoom enlarges the image size thereby reducing the effects due to pixel quantization. In addition, the rhombus mark must always remain in the field of view of the camera; this is most critical during the initial phase when the spacecraft undergoes a reorientation to be consistent with the desired docking orientation. This has provided our motivation for the use of a nonlinear observer-based controller for the spacecraft rotational control loops.

\section{Conclusions}

In this paper, we have proposed an automatic spacecraft docking control system using a computer vision system as relative position and orientation sensors. This docking control system compares favorably with other methods based on use of sensors such as laser, infrared, radar, GPS or inertial navigation.

Desired rotational and translational commands are easily specified for the spacecraft guidance and control system to achieve soft docking. We have proposed use of a computer vision system to estimate the relative position and orientation, and use of a nonlinear rotational observer and a linear translational observer to estimate their rates. The computer visionbased methods $s^{6,8}$ have advantages when compared with other relative position and orientation sensing methods. In particular, the estimation accuracy of the relative position and orientation estimates using a computer vision system improves as the range between the spacecraft and the space station decreases. Consequently, the control accuracy of the rotational and translational control loops improves as the range between the spacecraft and the space station decreases. Therefore, this computer vision-based control system is well suited for precise automatic spacecraft docking.

Major error sources in the proposed docking system, quantization measurement errors on the image plane, extrapolation and sampled data errors, and orbital effects have been considered in our simulations. Our experience indicates that these effects are easily compensated by the docking control system. It is important that the camera have an automatic, adaptive zoom to compensate for range effects. The ultimate accuracy of the integrated docking control system is then extremely good and depends primarily on the image-processing characteristics, namely the pixel quantization of the image plane. These results demonstrate the practical feasibility of the proposed docking system.

\section{References}

'Korbly, L., "Sensing Relative Attitudes for Automatic Docking," Journal of Guidance and Control, Vol. 6, No. 3, 1983, pp. 213-215.

${ }^{2}$ Niwa, S., Suzuki, M., and Kimura, K., "Electrical Shock Absorber for Docking System in Space," IEEE International Workshop on Intelligent Motion Control, Bogazici Univ., Istanbul, Turkey, 1990 , pp. 825-830.

${ }^{3}$ Olszewski, O. W., "Automated Terminal Guidance for a Shuttle Rendezvous to Space Station Freedom," Proceedings of the AIAA Guidance, Navigation, and Control Conference (Portland, OR), AIAA, Washington, DC, 1990, pp. 377-387.

${ }^{4}$ Tchoryk, P., et al., "Autonomous Rendezvous and Docking Using an Expendable Launch Vehicle," AIAA/Utah State Univ. Conf. on Small Satellites, 1991.

${ }^{5}$ Ho, C.-C. J., and McClamroch, N. H., "A Spacecraft Docking Problem: Postion Estimation Using a Computer Vision Approach," Proceedings of the AIAA Guidance, Navigation, and Control Conference (Portland, OR), AIAA, Washington, DC, 1990, pp. 1313-1318.

${ }^{6} \mathrm{Ho}$, C.-C. J., and McClamroch, N. H., "A Spacecraft Docking Problem: Position and Orientation Estimation Using a Computer Vision Approach," American Control Conf., Boston, MA, June 1991.

${ }^{7}$ Brody, A. R., "Evaluation of the ' $0.1 \%$ Rule' for Docking Maneuvers," Journal of Spacecraft and Rockets, Vol. 27, No. 1, 1990, pp. 7-8.

${ }^{8}$ Ho, C.-C. J., "Automatic Spacecraft Docking Using Computer Vision and Nonlinear Control Techniques," Ph.D. Dissertation, Dept. of Aerospace Engineering, Univ. of Michigan, Ann Arbor, MI, April 1991.

${ }^{9}$ Crouch, P. E., "Spacecraft Attitude Control and Stabilization: Applications of Geometric Control Theory to Rigid Body Models," IEEE Transactions on Automatic Control, Vol. 29, No. 4, 1984, pp. 321-331.

${ }^{10} \mathrm{Ho}$, C.-C. J., and McClamroch, N. H., "Guidance and Control of a Computer Vision Based Spacecraft Docking System," American Control Conf., Chicago, IL, June 1992. 